\title{
Contraintes et spécificités de l'évaluation clinique des dispositifs médicaux
}

\author{
François Parquin ${ }^{1}$, Antoine Audry ${ }^{2}$ et les participants à la table ronde $N^{\circ} 1$ de Giens XXVII * \\ 1 Hôpital Foch, Suresnes, France \\ 2 Medtronic France SAS, Boulogne, France
}

Texte reçu le 20 mars 2012 ; accepté le 4 juin 2012

\begin{abstract}
Mots clés :
liens d'intérêts ;

experts ;

impartialité

Résumé - L'objet de cette table ronde était de faire des propositions et recommandations concernant l'évaluation clinique des dispositifs médicaux (DM). Dans un premier temps, un rappel du parcours réglementaire tant au niveau européen que français est indispensable pour aborder ce champ encore jeune et très évolutif. Puis les principales spécificités des DM sont soulignées avec leurs implications sur l'évaluation clinique. Deux domaines, les DM implantables et les DM de compensation du handicap, sont plus particulièrement analysés pour les illustrer. Huit propositions et recommandations ont été retenues par la table ronde. Elles s'adressent à tous les acteurs du DM : industriels, utilisateurs, institutionnels et ont pour but d'améliorer une évaluation clinique centrée sur le patient et à son service.
\end{abstract}

Abréviations : voir en fin d'article.

\section{Introduction}

L'évaluation clinique des dispositifs médicaux (DM) couvre des champs extrêmement divers, ce qui impose de faire des choix en termes de périmètre et de perspective. Le travail réalisé dans le cadre de cette table ronde (TR) s'est ainsi volontairement centré sur l'évaluation clinique à réaliser dans l'intérêt du patient en essayant de dépasser les aspects réglementaires. Il a aussi pris le parti du retour d'expérience et d'expertise plutôt que celui d'une revue et analyse de la littérature existante sur le sujet (sans pour autant l'exclure totalement). Enfin, les solutions méthodologiques propres à chacune des situations traitées ont été volontairement écartées.

Après un premier travail d'inventaire réglementaire et des définitions existantes, le groupe a focalisé son attention sur quatre spécificités reconnues comme pouvant impacter directement les modalités d'acquisition des données cliniques, et sur deux secteurs dont les particularités en matière d'évaluation clinique complétaient utilement le premier niveau d'analyse thématique.

\section{Le contexte}

Deux caractéristiques sont de nature à impacter de façon significative la production de données cliniques et justifient à elles seules le choix de la thématique de cette table ronde :
- l'extrême diversité du monde des DM : la simple lecture de la définition réglementaire du dispositif médical issue de la directive 93/42/CE suffit à éclairer ce point : «On entend par dispositif médical tout instrument, appareil, équipement, matière, produit, à l'exception des produits d'origine humaine, ou autre article utilisé seul ou en association, y compris les accessoires et logiciels intervenant dans son fonctionnement, destiné par le fabricant à être utilisé chez l'homme à des fins médicales et dont l'action principale voulue n'est pas obtenue par des moyens pharmacologiques ou immunologiques ni par métabolisme, mais dont la fonction peut être assistée par de tels moyens »; [1]

- l'opérateur est multiple : professionnel de santé (médical et/ou, paramédical) ou le patient lui même, dans des environnements divers : hôpital, cabinet libéral, domicile du patient. Le DM peut être lié à un acte qui devra être évalué conjointement notamment dans le cas de DM innovant.

\section{1. Parcours réglementaire et définitions}

Le monde du DM est marqué par un environnement réglementaire jeune, dynamique et en perpétuelle évolution. À cela deux grandes conséquences :

- une connaissance souvent partielle des différentes étapes du parcours d'accès au marché (mise sur le marché et modalités de

\footnotetext{
* Pour la liste des participants, voir en fin d'article.
} 
prise en charge) alors même qu'elles jouent un rôle déterminant dans le développement de la preuve clinique. Ce chapitre en rappelle les principaux déterminants, leur analyse s'étant révélée très structurante dans la suite des débats de la TR ;

- un rythme d'évolution rapide : les évolutions se dessinent avant même que l'on ait eu le temps de mesurer l'impact des précédents changements. Le contenu de cet article devra donc être revu à l'aune des changements introduits dans le système.

\subsubsection{En France}

Deux grandes étapes existent et se succèdent, le cas échéant (tableau I). La prise en compte anticipée des besoins spécifiques à chacune de ces deux étapes peut permettre que certaines données produites pour la première soient exploitables pour la seconde.

L'autorisation de mise sur le marché : le marquage CE. Il s'agit d'une procédure européenne de mise sur le marché, pilotée par des organismes notifiés européens, accrédités par les autorités compétentes de chaque pays de l'Union européenne. Trois directives $^{[2,3,4]}$ constituent le socle réglementaire et définissent les exigences essentielles de performance et de sécurité auxquelles doivent se conformer les DM pour pouvoir être mis sur le marché européen. Elles sont complétées de normes et d'outils d'interprétation appelés Meddev, qui sont autant de référentiels sur lesquels les entreprises doivent s'appuyer pour démontrer la conformité de leur DM aux exigences essentielles.

Schématiquement, le marquage CE s'appuie sur trois procédés pour la démonstration de la conformité aux exigences de sécurité et de performance définie par ces directives : l'analyse de risque, l'évaluation pré-clinique (bancs d'essais, essais sur animaux...) et l'évaluation clinique. Ces trois éléments visent à démontrer que le rapport bénéfice / risque du dispositif concerné est favorable.

Ces dispositions ont été renforcées dans la directive 2007/47/CE ${ }^{[5]}$ qui s'impose désormais. Cette directive a permis en particulier de : - préciser ce que recouvre la terminologie « données cliniques »;

- rendre plus systématique la mise en place d'investigations cliniques spécifiques du DM concerné ; l'exigence est plus forte dans les domaines de risque les plus élevés (DM implantables et DM de classe III) pour lesquels, "les investigations cliniques doivent être réalisées, sauf si le recours aux données cliniques existantes peut être dûment justifié » ;

- systématiser les démarches de post-market surveillance visant à garantir la mise à jour régulière des données d'évaluation clinique : «L'évaluation clinique et sa documentation doivent être mises à jour activement au moyen des données obtenues par la surveillance après commercialisation. La décision de ne pas mener un suivi clinique dans le cadre du plan de surveillance du dispositif après commercialisation doit être dûment justifiée et documentée ».

La directive 2007-47/CE marque ainsi un vrai changement de paradigme en positionnant l'investigation clinique au cœur du processus de démonstration du bénéfice/risque et comme l'un des piliers de la gestion du rapport bénéfice/risque. Pour autant, d'application récente, ses effets sont encore difficilement mesurables.

L'obtention du remboursement : l'obtention du remboursement ${ }^{[6]}$ repose sur une évaluation scientifique et indépendante de la Commission nationale d'évaluation des dispositifs médicaux et technologies de santé (CNEDiMTS), commission de la Haute autorité de santé (HAS).

Schématiquement, un produit devra préalablement avoir démontré un service attendu (SA)/service rendu (SR) favorable. Il devra aussi démontrer le niveau d'amélioration du service attendu (ASA)/service rendu (ASR) qu'il apporte en regard des alternatives thérapeutiques/diagnostiques existantes.

L'analyse des essais cliniques constitue le pilier de la démonstration du SA/SR et de ASA/ASR. En matière de SA/SR l'intérêt du produit est apprécié au regard des données cliniques démontrant un rapport bénéfice/risque favorable et de la place du DM dans la stratégie thérapeutique/diagnostique. La notion d'ASA/ASR se fonde sur des études cliniques évaluant le dispositif par rapport au comparateur de référence dans la stratégie thérapeutique. La CNEDiMTS évalue, si besoin, l'acte associé, avec une même grille d'analyse.

Lors de son évaluation la CNEDiMTS précise les indications, la population cible correspondante, les conditions d'utilisation spécifiques le cas échéant, la durée d'inscription, les conditions de réinscription liées ou non à une étude post-inscription.

Le processus d'évaluation clinique est ici aussi un processus continu ayant vocation à être réévalué régulièrement ; il s'appuie sur la production de données cliniques en amont et en aval de la prise en charge.

\subsubsection{Qu'en est-il de la réglementation américaine d'autorisation de mise sur le marché ?}

Installée depuis plus longtemps que la réglementation $\mathrm{CE}$, la réglementation Food and Drug Administration (FDA) qui encadre la mise sur le marché des DM s'appuie sur des principes similaires à ceux qui régissent le cadre européen :

- classification des produits en fonction du risque associé à leur utilisation et définition de modes de preuves plus exigeants (cumulatifs) pour les classes de risque les plus élevées ;

- deux modes de preuve : le $510 \mathrm{k}$ et le PMA, ce dernier étant beaucoup plus exigeant et réservé aux produits de haut risque et/ ou n'ayant pas réellement d'équivalent sur le marché ;

- renforcement des exigences en matière de démonstration de la preuve clinique ;

- définition de mesures de dérogation (exemption) pour prendre en compte des situations très spécifiques (humanitarian device, substantial equivalence).

Sans doute la principale différence peut se résumer dans une prise en compte plus aiguisée du domaine thérapeutique considéré. Elle se concrétise par des critères spécifiques pour la classification 
Tableau I. Parcours du DM en France.

\begin{tabular}{|c|c|c|}
\hline Dispositifs médicaux & Médicaments & $\begin{array}{l}\text { Actes et technologies } \\
\text { de santé }\end{array}$ \\
\hline \multicolumn{3}{|c|}{ Études cliniques } \\
\hline Industrie & Industrie & CHU et professionnels de santé \\
\hline & Mise sur le marché & $=$ \\
\hline $\begin{array}{l}\text { Organisme notifié (marquage CE) } \\
\text { ANSM (autorité compétente) } \\
\text { Surveillance du marché }\end{array}$ & $\begin{array}{l}\qquad \text { EMA* }^{*} \text { ANSM } \\
\text { Evaluation de l'efficacité et de la sécurité } \\
\text { Commission européenne - ANSM : } \\
\text { Autorisation de mise sur le marché (AMM) }\end{array}$ & : \\
\hline \multicolumn{3}{|c|}{ Évaluation du service attendu / service médical rendu } \\
\hline HAS & HAS & $\mathrm{HAS}$ \\
\hline 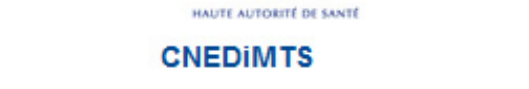 & $\begin{array}{l}\text { Commission de la } \\
\text { Transparence }\end{array}$ & 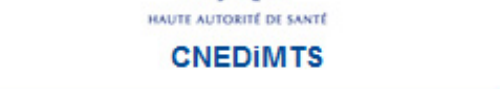 \\
\hline \multicolumn{3}{|c|}{ Fixation des prix / tarifs / taux } \\
\hline \multicolumn{2}{|c|}{$\begin{array}{ll}\text { médicaments et des dispositifs } & \cdot \text { taux de rembourseme } \\
\text { après négociation avec les industriels } & \cdot \text { tarifs et taux de remb }\end{array}$} & $\begin{array}{l}\text { UNCAM } \\
\text { ent des médicaments } \\
\text { oursement des actes après négociation } \\
\text { s des professionnels de santé }\end{array}$ \\
\hline \multicolumn{3}{|c|}{ Décision de prise en charge } \\
\hline \multicolumn{2}{|c|}{ Établit la liste des médicaments et des dispositifs médicaux remboursables } & $\begin{array}{l}\cdot \quad \text { UNCAM } \\
\text { Établit la liste des actes } \\
\text { remboursables }\end{array}$ \\
\hline
\end{tabular}

\section{HAS}

AMM : autorisation de mise sur le marché ; ANSM : Agence nationale de sécurité du médicament et des produits de santé (ex-Afssaps : Agence française de sécurité sanitaire des produits de santé) ; CEPS : Comité économique des produits de santé ; CNEDIMTS : Comission nationale d'évaluation des dispositifs médicaux et technologies de santé ; DM : dispositifs médicaux ; EMA : European Medicines Agency ; HAS : Haute autorité de santé ; UNCAM : Union nationale des caisses d'Assurance maladie.

des DM et par de nombreux guidelines spécifiques. Des réflexions et débats sont en cours aux USA pour les faire évoluer et pallier ainsi certaines limites.

\subsection{Clarifications}

À ce stade, il est intéressant de s'arrêter sur deux notions qui ont plus particulièrement marqué les débats au sein de la TR : la première a trait aux confusions de vocabulaire, la seconde au principe d'équivalence, élément particulièrement important dans le monde du DM.

\subsubsection{Points de vocabulaire}

C'est sans aucun doute l'une des premières conclusions de la TR : nombre de malentendus naissent d'une interprétation différente des termes par les uns et les autres au premier rang duquel figure la notion d'évaluation clinique.

- évaluation clinique : pour le marquage $\mathrm{CE}$, cette notion renvoie à l'existence de données cliniques pertinentes issues d'investigations cliniques sur le DM concerné ou d'autres sources ; en matière de prise en charge cette dimension renvoie aux données issues d'essais cliniques ;

- investigation clinique/essai clinique : étude spécifiquement menée sur le DM considéré ; 
- évidence clinique : somme des données pré-cliniques et cliniques existantes (propres au DM considéré ou pas) ;

- performance : effet revendiqué par le fabricant ;

- destination : "utilisation à laquelle un dispositif médical est destiné d'après les indications fournies par le fabricant dans l'étiquetage, la notice d'instruction ou les matériels promotionnels »; ${ }^{[7]}$ pour les produits à usage thérapeutique la destination est souvent précisée au travers d'indications.

\subsubsection{La notion d'équivalence}

Cette notion est essentielle et est reprise tant au plan du marquage $\mathrm{CE}^{[8]}$ que de la réglementation édictée par la FDA. ${ }^{[9]}$

Elle s'appuie sur le principe qu'une équivalence ne peut être revendiquée que dans le cas où le produit ne génère pas de risque additionnel ET est utilisé dans des indications et conditions strictement similaires au produit dont il revendique l'équivalence.

C'est pourquoi les réglementations retiennent trois dimensions différentes et cumulatives pour démontrer une telle équivalence :

- clinique : DM utilisé dans une destination (partie du corps, ...) et des indications similaires, pour une population cible similaire (âge, anatomie, physiologie) dans des conditions similaires ;

- technique : DM présentant des caractéristiques techniques (matériaux utilisés, viscosité, résistance, ...) et de conception similaires ; utilisant le cas échéant des techniques de pose et/ou opératoires similaires ;

- biologique : DM ayant des matériaux et état de surface en contact avec le corps humain (partie du corps, fluides) similaires.

Cette notion peut s'appliquer à de nombreux produits y compris des produits de haut risque, comme par exemple deux stimulateurs cardiaques simple chambre asservis.

Il n'est donc pas nécessaire d'être strictement identique pour être équivalent. Par contre, toute différence sur l'une de ces trois dimensions doit être justifiée au regard du rapport bénéfice/risque. Ces données peuvent être établies sur bancs d'essai, par des essais sur animaux, ou la production de données cliniques spécifiques. Pour autant, le sujet reste encore très complexe.

\section{Les contraintes et spécificités de l'évaluation clinique des DM}

Dans un premier temps quatre spécificités du DM ontété analysées de façon indépendante. Puis, deux secteurs particuliers, considérés comme emblématiques, ont été sélectionnés : les DM implantables (DMI) et les DM utilisés dans la compensation du handicap.

\subsection{Les spécificités du DM}

\subsubsection{Spécificité 1 : hétérogénéité du monde du DM}

Le bon sens seul suggère que face à l'extrême hétérogénéité des DM une réponse unique est impossible et ne serait pas reproductible.
Il faut donc considérer plusieurs situations et modes d'approches différents :

- prédictibilité de la performance : une canne, un gant, une compresse, un inlet dentaire, un pousse seringue électrique doivent satisfaire à des normes techniques, qui prédisent bien leur performance. Il n'y a donc pas d'intérêt a priori à mettre en œuvre des essais cliniques spécifiques. Ceci ne vaut toutefois que si les critères d'équivalence ne sont pas affectés (revendication, environnement clinique d'utilisation et caractéristiques techniques). Dès lors qu'une compresse revendiquerait une action ou une efficacité supplémentaire ou différente la question de la réalisation d'études cliniques devrait se poser ;

- niveau d'innovation du DM considéré : s'agit il d'une évolution incrémentale ou d'une évolution de rupture avec impact clinique élevé ? Dans les deux cas des essais cliniques seront nécessaires mais avec des objectifs requis différents et/ou adaptés (tableau II). Les mesures spécifiques d'accompagnement de l'innovation de rupture que ce soit au stade du développement préclinique ou à la phase précoce clinique n'ont pas été discutées lors de cette table ronde. Dans les autres cas, une question se pose : s'agit-il d'un DM similaire à un autre précédemment évalué. Cette notion apparemment simple, bien qu'explicitée par des textes européens (Meddev 2.7.1) ou américains (procédure $510 \mathrm{k}$ ) est en réalité complexe. Elle ne doit pas être affichée mais démontrée. Par exemple un acide hyaluronique à visée d'injection intra-articulaire dans le traitement de l'arthrose peut sembler similaire à un autre mais un degré de polymérisation plus ou moins élevé pourra entraîner des effets différents ou une durée d'action différente. La CNEDIMTS a ainsi estimé indispensable que tous les acides hyaluroniques revendiquant le statut de DM fournissent des études cliniques se comparant à des DM ou à des médicaments déjà évalués. On peut aussi prendre l'exemple des défibrillateurs cardiaques implantables. Il n'est pas nécessaire de démontrer pour chaque nouveau dispositif de cette catégorie l'intérêt clinique de la fonction première, à savoir délivrer un choc électrique interne dans le traitement de troubles du rythme ventriculaire ; le respect d'une série de spécifications techniques très précises et bien définies est nécessaire et suffisant. Par contre l'ajout d'un nouvel algorithme de délivrance du choc ou d'une nouvelle fonction doit déboucher sur une double démonstration : celle de son intérêt dans la stratégie thérapeutique et celle de l'absence d'effet négatif sur la fonction première du défibrillateur. Des études cliniques seront donc le plus souvent nécessaires. Le marquage CE prend en compte ce principe mais les informations qui y conduisent ne sont pas publiques, en particulier pour des raisons de «propriété industrielle ». Un compromis avec les fabricants doit être trouvé. Il pourra servir de base pour une démonstration plus complète visant à s'assurer que cette similarité ne s'accompagne pas de performance clinique ou d'effets secondaires différents ;

- catégorisation des DM : ainsi pour les stents coronaires des normes internationales et des recommandations HAS existent et 
Tableau II. Approche des DM selon leur degré d'innovation.

\begin{tabular}{|c|c|c|c|c|}
\hline \multirow{2}{*}{$\begin{array}{c}\text { Degré de } \\
\text { nouveauté } \\
\text { Degree of } \\
\text { novelty }\end{array}$} & \multirow{2}{*}{$\begin{array}{l}\text { Type de nouveauté } \\
\text { Type of novelty }\end{array}$} & \multicolumn{3}{|c|}{$\begin{array}{l}\text { Nouveauté à dominante } \\
\text { Innovation where the dominant is : }\end{array}$} \\
\hline & & $\begin{array}{l}\text { Technologique } \\
\text { Technological }\end{array}$ & & $\begin{array}{r}\text { Clinique } \\
\text { Clinical }\end{array}$ \\
\hline 5 & $\begin{array}{l}\text { Innovation majeure } \\
\text { Major innovation }\end{array}$ & $\begin{array}{l}\text { Rupture technologique } \\
\text { Breaking technology }\end{array}$ & $\begin{array}{l}\text { et } \\
\text { and }\end{array}$ & $\begin{array}{l}\text { Impact clinique fort } \\
\text { Strong clinical impact }\end{array}$ \\
\hline & $\begin{array}{c}\text { Innovation } \\
\text { (dispositif innovant) } \\
\text { (innovative device) }\end{array}$ & $\begin{array}{l}\text { Rupture technologique } \\
\text { Breaking technology }\end{array}$ & $\begin{array}{l}\text { ou } \\
\text { or }\end{array}$ & $\begin{array}{l}\text { Impact clinique fort } \\
\text { Strong clinical impact }\end{array}$ \\
\hline & $\begin{array}{l}\text { Nouveauté substantielle } \\
\text { Substantial novelty }\end{array}$ & $\begin{array}{l}\text { Incrémentation technique } \\
\text { Incremental technology }\end{array}$ & $\begin{array}{l}\text { et } \\
\text { and }\end{array}$ & $\begin{array}{l}\text { Impact clinique modéré } \\
\text { Moderate clinical impact }\end{array}$ \\
\hline & $\begin{array}{l}\text { Nouveauté modérée } \\
\text { Modarate novelty }\end{array}$ & $\begin{array}{l}\text { Incrémentation technique } \\
\text { Incremental technology }\end{array}$ & $\begin{array}{l}\text { ou } \\
\text { or }\end{array}$ & $\begin{array}{l}\text { Impact clinique modéré } \\
\text { Moderate clinical impact }\end{array}$ \\
\hline & $\begin{array}{l}\text { Nouveauté inexistante ou mineure } \\
\text { Lacking or minor novelty }\end{array}$ & $\begin{array}{l}\text { Technologie connue } \\
\text { Known technology }\end{array}$ & et & $\begin{array}{l}\text { Impact clinique inchangé } \\
\text { Unchanged clinical impact }\end{array}$ \\
\hline
\end{tabular}

DM : dispositifs médicaux

\section{Degré de nouveauté Degree of novelty}

précisent quel type d'étude est requis pour garantir le bon niveau d'évaluation clinique d'un nouveau stent. Cette approche est encore rarement utilisée car peu de référentiels sont actuellement disponibles. Elles sont indicatives à un moment donné en fonction des connaissances scientifiques disponibles. Les indications respectives des stents nus ou à élution de principe actif ont ainsi évolué depuis ces dernières années. Par ailleurs pour un même dispositif ces recommandations ne sont valables que dans une indication particulière. Ainsi, certains stents développés initialement pour la prise en charge de pathologies vasculaires artérielles périphériques peuvent aussi être utilisés en pathologie veineuse voire en en pathologie biliaire. Il faut évidemment refaire des études pour chaque indication.

Aucune de ces différentes approches ne peut être utilisée seule. Elles sont complémentaires et évolutives en fonction, notamment, du développement des connaissances scientifiques sur le thème considéré.

\subsubsection{Spécificité 2 : cycles de vie}

Certaines classes de DM évoluent très rapidement, selon un mode incrémental. La principale conséquence réside dans le fait que ces cycles de vie peuvent être plus courts que la durée des essais cliniques. L'analyse d'une étude réalisée avec des versions différentes est ainsi parfois quasi impossible. La constitution de sous groupes a posteriori ne peut être recommandée. Même si quelques réponses méthodologiques existent, il est préférable d'éviter de telles situations en anticipant dans le schéma initial de l'étude l'apparition d'une évolution incrémentale souvent prévisible. Un comité scientifique indépendant doit pouvoir prendre en compte une évolution incrémentale mineure dans une étude. Le point essentiel est donc de discriminer ce qui, dans la nouvelle génération peut impacter les résultats de l'évaluation clinique en cours et d'en tenir compte dans l'analyse statistique. En calculant bien les effectifs et le nombre de centres nécessaires, en favorisant une mise en œuvre rapide des études, on évitera plus facilement ce type de situation. L'utilisation de critères intermédiaires prédictifs de l'efficacité clinique a aussi été l'une des approches discutées au sein de la TR pour permettre de rapprocher cycles de vie et durée d'évaluation.

Une évolution incrémentale conduisant à une nouvelle référence doit faire l'objet d'étude spécifique dès lors qu'elle impacte le rapport bénéfice/risque. Par exemple, on peut modifier le dispositif d'ancrage d'une prothèse endovasculaire ce qui pourra diminuer le risque d'endofuite, mais il ne doit pas y avoir plus de risque hémorragique à la pose. 
À l'opposé, la vie d'un dispositif peut être très longue chez un patient. Il en est ainsi par exemple pour nombre de DMI implantés chez l'enfant ou l'adulte jeune, prothèses orthopédiques ou auditives implantables... Dans ce cas, le suivi à long terme est fondamental ; des études de longue durée devant démontrer le maintien de l'efficacité.

\subsubsection{Spécificité 3 : caractère opérateur dépendant}

Le caractère opérateur dépendant est un élément fondamental et constitutif de l'utilisation des DM. Une prothèse de hanche a ses qualités propres et ses indications. Mais l'expérience du chirurgien, sa bonne connaissance du dispositif, le choix de la voie d'abord adaptée, une bonne préparation de la zone d'implantation seront évidemment déterminants pour obtenir l'efficacité recherchée. De même, certains effets secondaires relèvent plus de la procédure et de l'opérateur que du DM lui même : risque hémorragique, ou infectieux par exemple. Dans certains cas, l'opérateur peut être le patient lui même (dispositifs d'auto-surveillance glycémique chez le patient diabétique, véhicules pour handicapés...) ce qui nécessite une formation technique spécifique.

De cette caractéristique, découle naturellement la notion de courbe d'apprentissage. Il faut en tenir compte à tous les stades du développement pré clinique, clinique et dans l'utilisation quotidienne du DM après sa mise sur le marché, en fournissant par exemple :

- la description très précise de la technique de pose ou de l'utilisation, le développement d'ancillaires adaptés et souvent spécifiques :

- la mise en place de formation à la technique, pré-requis à l'utilisation du dispositif médical et partie intégrante du plan de gestion des risques. Des bancs d'essais et des simulateurs sont de plus en plus utilisés dans ce cadre pour faciliter l'acquisition de pratiques interventionnelles ;

- la prise en compte des retours d'expérience pour définir de premières évolutions incrémentales aussi bien sur le DM lui même que sur d'éventuels ancillaires.

Quoiqu'il en soit les essais devront intégrer cette courbe d'apprentissage en prévoyant une première phase d'acquisition, dans le nombre de sujets nécessaires par exemple, et/ou par d'éventuelles analyses intermédiaires. Il sera nécessaire aussi dans le cadre d'études post-mise sur le marché de suivre l'impact de la courbe d'apprentissage sur l'utilisation du DM et d'en déduire les conditions environnementales propres à une diffusion efficace de la technique.

\subsubsection{Spécificité 4 : petites populations}

Cette situation est très fréquente dans le domaine du DM. Par exemple la population cible d'un système d'aérosolthérapie destinée au traitement des patients atteints de mucoviscidose est de 4500 patients, celle d'une prothèse de genou monoaxiale commandée par microprocesseur est de 1200 à 1400 patients, celle des stents coronaires à élution de produit actifs de 65000 patients environ. On est bien loin de la population cible d'un médicament anti hypertenseur et plus souvent dans des niveaux de population qui relèveraient de la notion de spécialité pharmaceutique orpheline. Ce phénomène est particulièrement fréquent dans le domaine du handicap ou des DM sur mesure. Les patients concernés sont dispersés sur l'ensemble du territoire et pris en charge dans de nombreuses équipes, ce qui réduit d'autant le nombre de patients suivis par équipe. La notion de centre de référence n'est ici que très rarement applicable. Des solutions notamment méthodologiques peuvent être proposées : réalisation d'essais cliniques au niveau européen par exemple ou recensement exhaustif des patients traités. Des systèmes facilitateurs sont en développement, en particulier au niveau européen.

\subsection{Deux secteurs emblématiques: DM implantables et DM du handicap}

\subsubsection{Secteur 1 : les DM implantables}

L'évaluation du bénéfice/risque dans le temps est au cœur de la problématique posée par le DMI. Dans le domaine pédiatrique par exemple, une prothèse valvulaire mécanique posée chez un adulte jeune, aura une durée de vie très longue. Des critères de jugement intermédiaires robustes, prédictifs de l'évolution à long terme sont souvent difficiles à déterminer. Des modélisations à partir de banc d'essai sont une aide utile en particulier pour déterminer les effets de l'usure des matériaux employés et de leur structure. Par exemple, des critères radiologiques sont proposés pour prédire l'usure des prothèses de hanche et essayer de prévoir les risques de descellement prothétiques. Des critères angiographiques ont été largement utilisés pour essayer de déterminer la resténose intrastent coronaire mais ils ne disent pas grand chose sur le risque de thrombose du stent à distance. Pour d'autres dispositifs tels les implants auditifs, il existe des pannes « floues », qui ne sont pas en tout ou rien. Si la panne est progressive, la personne implantée s'y habitue, comme par exemple lors de l'encrassement d'un microphone. La sanction pour l'implanté est la défaillance des performances qu'il met sur le compte de ses capacités personnelles. Il est donc nécessaire de suivre les patients implantés tout au long de leur vie.

Il faut aussi prendre en compte l'obsolescence d'un DM. Audelà de la notion de garantie, comment assurer le «service après vente » d'un DMI implanté chez un enfant, alors que la technologie évolue?

Doivent également être pris en compte certaines particularités des DMI comme la difficulté du double aveugle, le caractère opérateur dépendant. 
Une part de la solution passe par la réconciliation des deux approches que sont l'évaluation pour la mise sur le marché, souvent restreinte en terme de suivi ou s'appuyant sur des critères intermédiaires, et la surveillance post-mise sur le marché qui doit démontrer le maintien de la performance du DM et sa fiabilité en se basant éventuellement sur des registres représentatifs de la population suivie. En France, la pratique des registres est peu développée. Elle gagnerait à être favorisée afin de mieux anticiper et de mieux surveiller la balance bénéfice/risques tout au long de la vie du DM.

\subsubsection{Secteur 2 : les dispositifs de compensation du handicap}

Ce champ est très vaste et d'analyse complexe. Les DM y sont très divers, ont des cycles de vie très variables, sont souvent opérateurs dépendants, et concernent de petites populations réparties sur l'ensemble du territoire national. Mais il faut pour aborder ce domaine essayer de bien comprendre ce qu'est le handicap. Commençons par rappeler la définition du handicap, issue de la loi pour « l'égalité des droits et des chances » de 2005 : «Constitue un handicap, toute limitation d'activité ou restriction de participation à la vie en société subie dans son environnement par une personne en raison de son altération substantielle, durable ou définitive d'une ou plusieurs fonctions physiques, sensorielles, mentales, cognitives ou psychiques, d'un polyhandicap ou d'un trouble de santé invalidant $\gg .{ }^{[10]}$ Cette même loi dans son article 11 précise que la personne handicapée a droit à la compensation des conséquences de son handicap et fait de l'élaboration d'un projet de vie la base de cette compensation. Ensuite, le handicap doit être considéré sous plusieurs angles complémentaires :

- maladie/trouble, déficience (par exemple handicapé par un déficit de l'audition);

- incapacité (handicapé pour entendre des signaux sonores) ;

- participation (handicapé si le travail comporte des informations transmises par signaux sonores).

La classification internationale du fonctionnement du handicap et de la santé (CIF) est aussi très utilisée. Elle permet de quantifier grâce à une échelle commune à 5 niveaux toutes les composantes : fonctions organiques, structures anatomiques, activité, participation et facteurs environnementaux.

Il importera aussi de déterminer le statut du produit considéré : produit d'assistance ou DM.

Ainsi, en matière de DM du handicap, l'évaluation est naturellement bipolaire. Elle comporte une partie technique soumise au respect de normes et une partie clinique qui sera obligatoirement centrée sur les besoins de la personne handicapée et de son projet de vie. Malgré ces particularités, les études cliniques sont possibles. Il faudra évaluer l'efficacité par l'amélioration de la déficience, de la capacité de réalisation et de la participation. La méthodologie des essais doit être adaptée et les objectifs bien identifiés. Vu la faible taille de la population cible, il sera parfois nécessaire de recourir à des études multicentriques internationales, seules capables de répondre rapidement aux questions posées. Un plus large recours aux critères de qualité de vie globale, parfois personnalisé, est indispensable.

\section{Les propositions et recommandations finales de la TR}

\subsection{Clarifier la terminologie de certains termes: évaluation clinique, essai clinique, performance}

Au travers des discussions et débats, il est très vite apparu que les terminologies en matière d'évaluation pouvaient revêtir des significations différentes selon que l'on faisait appel à des textes relevant du domaine européen du marquage $\mathrm{CE}$ ou du domaine national de la prise en charge. Dès lors que ces barrières sont levées, la discussion peut s'appuyer sur un socle commun. Cette action pourrait être mise en œuvre par les agences en charge de ces sujets qui diffuseraient largement alors une forme de lexique propre aux DM et à leur évaluation clinique, valide quelle que soit la perspective choisie.

\subsection{L'évaluation clinique doit permettre d'éclairer le bénéfice/risque, les indications, la place dans la stratégie thérapeutique et la population cible}

Cette perspective a le mérite de permettre la synthèse entre les besoins des différentes parties prenantes au système de soins : le patient reste l'élément central, mais aussi l'opérateur et celui qui a en charge de s'assurer que les deniers de l'État sont utilisés à bon escient et dans des conditions optimales.

\subsection{Les essais cliniques ne sont pas toujours appropriés}

La nature même de certains dispositifs médicaux (mécanique, physique...) rend leur évaluation plus modélisable sur bancs d'essais du fait d'une prédictibilité plus forte de leur comportement en situation clinique et de la moindre variabilité de leurs performances d'une personne à l'autre. Par ailleurs, le processus de développement incrémental des DM soulève la difficile question de l'équivalence. Les efforts d'ores et déjà entrepris tant au plan européen qu'au plan américain doivent être poursuivis afin de décrire les méthodes permettant de garantir le meilleur niveau de démonstration de cette équivalence. Les résultats doivent en outre être mieux communiqués. Ainsi pourra-t-on introduire un niveau de confiance élevé, seul facteur susceptible de concourir à faire reconnaître la justification du non recours aux essais cliniques pour démontrer le rapport bénéfice/risque de certains DM. 


\subsection{Les données du marquage CE doivent être plus accessibles et transparentes dans plusieurs domaines: plan de gestion risque, équivalence, élaboration du résumé des caractéristiques du dispositif}

Les données restent encore trop souvent cantonnées aux dossiers de marquage $\mathrm{CE}$ et sont très rarement communiquées tant aux autorités sanitaires (à titre proactif) et encore moins aux professionnels de santé (utilisateurs-patients ou personnels de santé-, gestionnaires...). Des prémices existent désormais dans le domaine des produits très innovants sous l'impulsion en particulier de la cellule innovation de l'ancienne Agence française de sécurité sanitaire des produits de santé (ex-Afssaps [désormais Agence nationale de sécurité du médicament et des produits de santé ou ANSM]) et plus récemment de programmes pilotés par la HAS : dans ce cadre un échange peut se mettre en place très en amont et permettre ainsi, au plan local, une communication plus large et plus structurée des différentes données d'évaluation existantes. Il est certain que tout le monde tirerait bénéfice de l'existence d'un résumé des caractéristiques du DM incluant notamment les données d'évaluation soutenant les revendications mises en avant pour le DM dans le cadre du dossier de marquage CE.

\subsection{Investir sur le suivi post-commercialisation à long terme, en particulier dans le champ des DMI}

Le rôle de l'évaluation en phase pré-mise sur le marché est essentiel pour garantir un niveau de service attendu suffisant tant au plan du bénéfice que des risques associés. Il est apparu tout aussi essentiel d'investir dans de nouveaux outils et de nouvelles méthodes de suivi post-mise sur le marché. Des initiatives ont vu le jour tant au plan européen (post-market clinical follow up) qu'au niveau français en matière de prise en charge (études post inscriptions). Dans ces deux axes, il est essentiel de poursuivre les travaux, de développer les méthodes les plus appropriées et de les faire, tant que faire se peut, converger pour garantir le développement d'une base de données utile et représentative. Des efforts doivent aussi être faits pour permettre l'utilisation des bases de données existantes (programme de médicalisation des systèmes d'information [PMSI], système national d'informations interrégimes de l'Assurance maladie [SNIIRAM]) dans un objectif de suivi des DM.

\subsection{Sensibiliser les utilisateurs à l'évaluation des DM}

Toute personne confrontée à la mise en place d'essais cliniques sait combien les méthodes et modalités habituelles (mises en œuvre dans d'autres domaines comme le médicament) doivent être adaptées pour tenir compte des spécificités des DM. Des solutions existent et doivent être privilégiées pour garantir le meilleur niveau de preuve possible pour chaque catégorie de DM. L'intensification d'actions de sensibilisation et de formation permettrait de créer et fonder une culture commune à tous les acteurs du circuit de l'évaluation. Elle pourrait être conduite conjointement entre les acteurs de terrain et les agences concernées (ex-Afssaps [désormais ANSM], HAS), en s'appuyant sur une documentation de référence, utilisable par tous et la programmation d'actions de sensibilisation via les filières déjà existantes (comité de protection des personnes [CPP], département recherche clinique et innovations [DRCI], établissement public à caractère industriel et commercial [OSEO], Pôles de compétitivité, Aviesan, Alliance pour la recheche et l'innovation des industries de santé [ARIIS], Sociétés savantes...).

\subsection{Approfondir les interactions CPP - monde du DM}

Il est nécessaire de poursuivre les travaux communs avec les CPP pour mieux diffuser les spécificités de l'évaluation du DM, et échanger sur les avancées en matière de méthodologie propre au DM.

\subsection{Adapter, optimiser les programmes de soutien à l'évaluation existants (programme de soutien aux techniques innovantes [STIC], programme hospitalier de recherche clinique $[\mathrm{PHRC}] \ldots$ )}

Une réforme de ces programmes a récemment vu le jour. Un bilan permettra de mesurer si ces évolutions répondent bien aux objectifs précisés lors de la TR.

\section{Remerciements}

Les auteurs remercient beaucoup tous les participants pour leur implication et leur contribution aux travaux de cette table ronde et plus particulièrement Anne Josseran pour son soutien logistique et ses conseils avisés.

\section{Participants.}

Antoine Audry (Medtronic France SAS), Bernard Avouac (Laser), Alexandre Barna (AP-HP, CEDIT), Catherine Denis (HAS, CNEDiMTS), Bruno Frachet (AP-HP, Hôpital Avicenne), Isabelle Fontes (Abbott Vascular), Chrystelle Gastaldi (Ministère de la santé, Direction sécurité sociale), Jean-Claude Ghislain (ANSM, DEDIM), Bernard Guillot (CHU Montpellier), Muriel Granger (Boston Scientific), Anne Grumblat (CHU Besançon), Anne Josseran (SNITEM), Gérard Luzergues (Ceiso), Pierre-Olivier Marguet (Hospices Civils Lyon, DRCI), Noël Martinet (CHU Nancy), Nadine Normand (AFNOR), François Parquin (Hôpital Foch), Rémy Pécault(CNAMTS), Sandrine Pitel(Qualissima), Françoise Roca (3M), Fabrice Romano (Eye Tech Care), Emilie Rufach (GMED), Dominique Thiveaud (Europharmat), Daniel Vasmant (ministère de l'Économie, des Finances et de l'Emploi, DGCIS). 


\section{Conflits d'intérêts. Aucun.}

Abréviations. ANSM : Agence nationale de sécurité du médicament et des produits de santé (ex-Afssaps [Agence française de sécurité sanitaire des produits de santé]); ARIIS : Alliance pour la recherche et l'innovation des industries de santé ; ASA : amélioration du service attendu; ASR : amélioration du service rendu ; CNEDIMTS : Commission nationale d'évaluation des dispositifs médicaux et technologies de santé ; CPP : Comité de protection des personnes; DM : dispositifs médicaux; DMI : dispositifs médicaux implantables; DRCI : Département recherche clinique et innovations ; FDA : Food and Drug Administration; HAS : Haute autorité de santé : OSEO : établissement public à caractère industriel et commercial; PHRC: programme hospitalier de recherche clinique ; PMSI : programme de médicalisation des systèmes d'information ; RCD : résumé des caractéristiques du dispositif ; SA : service attendu ; SNIIRAM : Système national d'informations inter-régimes de l'Assurance maladie; SR: service rendu ; STIC : programme de soutien aux techniques innovantes ; TR : table ronde.

\section{Références}

1. Article L665-3 du Code de la Santé Publique. http://www.legifrance.gouv.fr/affichCodeArticle.do?cidTexte= LEGITEXT000006072665\&idArticle=LEGIARTI000006694037\&dateTexte $=20120311$

2. Directive européenne 90/385/CE (dispositifs médicaux implantables actifs), 1990. http://eur-lex.europa.eu/Notice.do?val=161305:cs\&lang=fr\&list= 217630:cs, 201866:cs,161305:cs,\&pos=3\&page=1\&nbl=3\&pgs=10\&hwords $=\&$ checktexte $=$ checkbox $\&$ visu $=\#$ texte
3. Directive européenne 93/42/CE (dispositifs médicaux), 1993. http://eurlex.europa.eu/Notice.do?val=294514: cs\&lang=fr\&list=335843:cs,329393: cs,329392:cs,317994:cs,294514:cs,\&pos=5\&page=1\&nbl=5\&pgs=10\&hwo rds $=\&$ checktexte $=$ checkbox $\&$ visu=\#texte

4. Directive européenne 98/79/CE (dispositifs de diagnostic in vitro), 1998 http://eur-lex.europa.eu/Notice.do?val=226562: cs\&lang=fr\&list=272230: cs,236122:cs,234415:cs,234414:cs,226562:cs,\&pos=5\&page=1\&nbl=5\&pg $\mathrm{s}=10 \&$ hwords $=$ \&checktexte=checkbox\&visu=\#texte

5. Directive européenne 2007/47/CE, 2007. http://eur-lex.europa.eu/ Notice.do?val $=455903: \mathrm{cs} \&$ lang $=$ fr\&list $=491391: \mathrm{cs}, 459453: \mathrm{cs}, 459452: \mathrm{cs}, 45$ 5903:cs, \&pos=4\&page=1\&nbl=4\&pgs=10\&hwords $=\&$ checktexte=checkbox\&visu=\#texte

6. Parcours du dispositif médical - Guide HAS, 2009. http://www.has-sante.fr/ portail/upload/docs/application/pdf/2009-12/presentation_guide_dm.pdf

7. Art R5211-4, http://www.legifrance.gouv.fr

8. Meddev 2.7.1, 2009. http://ec.europa.eu/health/medical-devices/files/ meddev/2_7_1rev_3_en.pdf

9. FDA guidance: substantial equivalence to a US marketed predicate device http://www.fda.gov/medicaldevices/deviceregulationandguidance/howtomarketyourdevice/premarketsubmissions/premarketnotification510k/ default.htm

10. Article L.114 du code de l'action sociale et des familles, loi pour l'égalité des droits et des chances, la participation et la citoyenneté des personnes handicapées. 11 février 2005. http://www.legifrance.gouv.fr/affichCodeArticle.do?cidTexte=LEGITEXT000006074069\&idArticle=LEGIARTI000006796 446\&dateTexte $=20111028$

Correspondance et offprints : François Parquin, Unité de Soins Intensifs Respiratoires, Hôpital Foch, BP 36, 92151 Suresnes Cedex, France.

E-mail : f.parquin@hopital-foch.org 